INPLASY

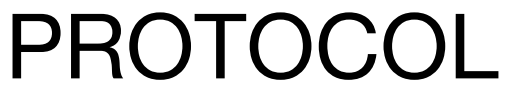

To cite: Guo et al. Effect of treatments on skin microbiota in patients with atopic dermatitis: a protocol for systematic review. Inplasy protocol 202140008. doi: 10.37766/inplasy2021.4.0008

Received: 02 April 2021

Published: 02 April 2021

Corresponding author: Bo Yu

yub_pkusz@126.com

Author Affiliation:

Peking University Shenzhen Hospital

Support: None.

Review Stage at time of this submission: Preliminary searches.

Conflicts of interest: None declared.

\section{Effect of treatments on skin microbiota in patients with atopic dermatitis: a protocol for systematic review}

Guo, Y1; Zhang, K2; Jian, X3; Dou, X'; Yu, B5 .

Review question / Objective: Review question: effect of treatments on skin microbiota in patients with atopic dermatitis; 1) Participants/population: patients with atopic dermatitis; 2) Interventions: treatments (including topical therapy, phototherapy, systemic treatment, etc.); 3) Comparators/ control: 1) before v.s. after treatment; 2) treatment v.s. placebo; 3) comparison between different types of treatment; 4) Outcomes: 1) primary outcomes: changes of skin microbiota diversity and differential microbes; 2) secondary outcomes: immunological factors, microbial interactions, and microbiota functions; 5) Study design: interventional research.

Condition being studied: Atopic dermatitis is a common chronic inflammatory skin disease, with a prevalence of $20 \%$ in children and $10 \%$ in adults. Atopic dermatitis is characterized by intense itch, recurrent eczematous lesions, and a fluctuating course. Several pathophysiological mechanisms contribute to atopic dermatitis etiology, including impairment of epidermal barrier function, type 2 immune responses, dysbiosis of the skin microbiota, etc. Current treatments for atopic dermatitis include topical therapy, phototherapy, systemic treatment, etc.

INPLASY registration number: This protocol was registered with the International Platform of Registered Systematic Review and Meta-Analysis Protocols (INPLASY) on 02 April 2021 and was last updated on 02 April 2021 (registration number INPLASY202140008).

\section{INTRODUCTION}

Review question / Objective: Review question: effect of treatments on skin microbiota in patients with atopic dermatitis; 1) Participants/population: patients with atopic dermatitis; 2) Interventions: treatments (including topical therapy, phototherapy, systemic treatment, etc.); 3) Comparators/ control: 1) before v.s. after treatment; 2) treatment v.s. placebo; 3) comparison between different types of 
treatment; 4) Outcomes: 1) primary outcomes: changes of skin microbiota diversity and differential microbes; 2) secondary outcomes: immunological factors, microbial interactions, and microbiota functions; 5) Study design: interventional research.

Rationale: Atopic dermatitis is a common chronic inflammatory skin disease characterized by recurrent eczematous lesions and intense itch, with a prevalence of $20 \%$ in children and $10 \%$ in adults. Human skin is an epithelial barrier to the external environment and supports diverse microbiota comprised of bacteria, fungi, viruses, etc. Skin microbiota dysbiosis showed an important role in the pathogenesis of atopic dermatitis, such as low diversity, high abundance of Staphylococcus aureus, etc. However, the effect of treatments on skin microbiota in atopic dermatitis remains unclear. Furthermore, to our knowledge, there is no systematic review to evaluate the effect of treatments on skin microbiota in patients with atopic dermatitis. It is therefore warranted to summarize the available literatures for understanding the role of skin microbiota in the treatment and prognosis of atopic dermatitis. Our systematic review may provide insights into etiology study and new targeted therapies of atopic dermatitis.

Condition being studied: Atopic dermatitis is a common chronic inflammatory skin disease, with a prevalence of $20 \%$ in children and $10 \%$ in adults. Atopic dermatitis is characterized by intense itch, recurrent eczematous lesions, and a fluctuating course. Several pathophysiological mechanisms contribute to atopic dermatitis etiology, including impairment of epidermal barrier function, type 2 immune responses, dysbiosis of the skin microbiota, etc. Current treatments for atopic dermatitis include topical therapy, phototherapy, systemic treatment, etc.

\section{METHODS}

Search strategy: The literature search strategy was a combination of search terms of "skin", "microbiota", and "atopic dermatitis". The literature search will be performed in PubMed, EMBASE, Web of Science, ClinicalTrials.gov, Chinese Clinical Trial Registry, etc.

Participant or population: Patients with atopic dermatitis.

Intervention: Treatments for atopic dermatitis (including topical therapy, phototherapy, systemic treatment, etc.).

Comparator: 1) before v.s. after treatment; 2) treatment v.s. placebo; 3) comparison between different types of treatment.

Study designs to be included: Interventional research (including randomized trials and non-randomized studies).

Eligibility criteria: We will include interventional studies (including topical therapy, phototherapy, systemic treatment, etc.) enrolled patients with atopic dermatitis and with skin microbiota measured. Inclusion criteria: 1) study design: Interventional research (including randomized trials and non-randomized studies); 2) sample type: skin microbiota samples (e.g. cutaneous swab samples); 3) restriction of study type: human studies.

Information sources: 1) Electronic databases: PubMed, EMBASE, Web of Science; 2) Trial registers: ClinicalTrials.gov, Chinese Clinical Trial Registry; 3) Contact with authors.

Main outcome(s): Primary outcomes: changes of skin microbiota diversity (alpha diversity and beta diversity) and differential microbes. Alpha diversity is a quantitative measure of community diversity and beta diversity is a measure of similarity between samples. Detection of differential microbes between groups is an exploration of key taxa, which could be potential biomarkers.

Additional outcome(s): Secondary outcomes: immunological factors, microbial interactions, and microbiota functions. 
Data management: Using pre-designed standardized data abstraction forms, two researchers independently extracted data, with any disagreements resolved by consensus. We will extract information of basic characteristics (e.g. authors, publication year, journal, etc.) and major findings of included studies.

Quality assessment / Risk of bias analysis: The following tools will be used for randomized trials and non-randomized studies: 1) Cochrane risk-of-bias tool for randomized trials (RoB 2) ; 2) Risk Of Bias In Non-Randomized Studies - of Interventions (ROBINS-I).

Strategy of data synthesis: We will provide a narrative synthesis of the findings from the included studies in terms of primary outcomes (alpha diversity, beta diversity, and differential microbes) and secondary outcomes (immunological factors, microbial interactions, and microbiota functions). However, meta-analysis is not planned as no specific effect measures (e.g. ORs, HRs) were reported in such studies focusing on microbiota.

Subgroup analysis: Subgroup analyses will be performed according to different types of treatment for atopic dermatitis, including topical therapy, phototherapy, systemic treatment, etc.

Sensitivity analysis: We consider sensitivity analysis for methodological quality by excluding the studies with low quality.

Country(ies) involved: China.

Keywords: Atopic dermatitis; Microbiota; Skin; Diversity; Staphylococcus aureus.

Contributions of each author:

Author 1 - Yang Guo - Literature search, study selection, quality assessment, and writing the original draft.

Author 2 - Kao-yuan Zhang - Literature search, study selection, and data extraction.

Author 3 - Xing-ling Jian - Data extraction and quality assessment.
Author 4 - Xia Dou - Methodology, review, and editing.

Author 5 - Bo Yu - Methodology, review, and supervision. 